\title{
OBSTRUCTIONS TO CHOOSING DISTINCT POINTS ON CUBIC PLANE CURVES
}

\author{
WEIYAN CHEN
}

\begin{abstract}
Every smooth cubic plane curve has 9 inflection points, 27 sextatic points, and 72 "points of type nine". Motivated by these classical algebro-geometric constructions, we study the following topological question: Is it possible to continuously choose $n$ distinct unordered points on each smooth cubic plane curve for a natural number $n$ ? This question is equivalent to asking if certain fiber bundle admits a continuous section or not. We prove that the answer is no when $n$ is not a multiple of 9. Our result resolves a conjecture of Benson Farb.
\end{abstract}

\section{INTRODUCTION}

A cubic plane curve in $\mathbb{C} P^{2}$ is the zero locus of a homogeneous polynomial $f(x, y, z)$ of degree 3. It has been a classical topic to study certain special points on smooth cubic curves, such as the 9 inflection points (dating back at least to Maclaurin; see the Introduction in [1] for an account of the history of this topic), the 27 sextatic points (studied by Cayley [2]), and the 72 "points of type 9" (studied by Gattazzo [5]). Inspired by these classical constructions, Benson Farb (private communication) asked the following question: For what integer $n$ is it possible to continuously choose $n$ distinct points on each cubic plane curve as the curve varies in families?

To make the question precise, let $\mathcal{X}$ denote the parameter space of smooth cubic plane curves:

$$
\mathcal{X}:=\{f(x, y, z): f \text { is a homogeneous polynomial of degree } 3 \text { and is smooth }\} / \sim
$$

where $f \sim \lambda f$ for any $\lambda \in \mathbb{C}^{\times}$. There is a fiber bundle whose fiber over $f \in \mathcal{X}$ is $\operatorname{UConf}^{n} C_{f}$, the configuration space of $n$ distinct unordered points on the cubic plane curve $C_{f}$ defined by $f=0$.

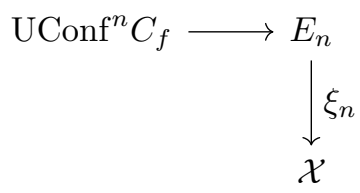

Question 1 (Farb). For which values $n$ do the bundles $\xi_{n}$ admit continuous sections?

The aforementioned algebraic constructions and their generalizations give continuous sections to $\xi_{n}$ for various $n$ :

Theorem 1 (Maclaurin, Cayley, Gattazzo). The bundle $\xi_{n}$ has a continuous section when $n=$ $9 \sum_{k \in S} J_{2}(k)$ where $J_{2}$ is Jordan's 2-totient function and $S$ is an arbitrary finite set of positive integers, for example, when $n=9,27,36,72,81,99,108,117,135,144,180 \ldots$

See Section 2 for more discussion on Theorem 1 and a definition of Jordan's 2-totient function.

In contrast to the various algebraic constructions of sections of $\xi_{n}$, it was previously unknown whether there is any $n$ such that $\xi_{n}$ does not have any continuous section.

Conjecture 1 (Farb). $n=9$ is the smallest value for $\xi_{n}$ to have a continuous section.

Behind this conjecture is the following speculation: the classical algebraic constructions should be the only possible continuous sections. The minimal number comes from the 9 inflection points. 
WEIYAN CHEN

After Conjecture 1, Farb made a much stronger conjecture: the only continuous sections of $\xi_{n}$ (up to homotopy) are those given by the algebraic constructions in Theorem 1.

We will prove Conjecture 1 in this paper. In fact, we will prove the following stronger statement.

Theorem 2. $\xi_{n}$ has no continuous section unless $n$ is a multiple of 9.

Let us remark on the significance of Theorem 2, it tells us that in those case when $9 \nmid n$ and when our current knowledge since Maclaurin had failed to identify any natural structure of $n$ special points on smooth cubic curves, there is in fact none.

Notice that $\xi_{1}$ is precisely the tautological bundle whose fiber over every curve in $\mathcal{X}$ is the curve itself. Theorem 2 thus implies:

Corollary 3. The tautological bundle $\xi_{1}$ does not have any continuous section.

Corollary 3 has the following interpretation: it is not possible to continuously choose an elliptic curve structure for every cubic plane curve $C_{f}$ in the parameter space $\mathcal{X}$, because it is not possible to continuously choose a point on $C_{f}$ to serve as the identity.

Between the known algebraic constructions (Theorem 1) and the topological obstructions presented here (Theorem 2), the smallest $n$ for which we don't know whether a continuous section to $\xi_{n}$ exists or not is $n=18$.

\section{ACKNOWLEDGEMENT}

I am grateful to Benson Farb for sharing his extremely interesting question and conjecture. I thank Igor Dolgachev for pointing me to the papers by Cayley and Gattazzo, and thank Ronno Das for making me aware of a mistake in the proof of Lemma 7 in an early draft.

\section{BACKGROUND}

2.1. The bundle $\xi_{n}$. We will first give more details about the fiber bundle $\xi_{n}$. Let PConf $^{n} C_{f}$ denote the configuration space of $n$ distinct ordered points on $C_{f}$ :

$$
\mathrm{PConf}^{n} C_{f}:=\left\{\left(x_{1}, x_{2}, \cdots, x_{n}\right) \in\left(C_{f}\right)^{n}: x_{i} \neq x_{j} \forall i \neq j\right\} .
$$

Define

$$
\widetilde{E_{n}}:=\left\{\left(f, x_{1}, x_{2}, \cdots, x_{n}\right) \in \mathcal{X} \times\left(\mathbb{C} P^{2}\right)^{n}: x_{i} \in C_{f} \forall i \text { and } x_{i} \neq x_{j} \forall i \neq j\right\} .
$$

The projection $\widetilde{E_{n}} \rightarrow \mathcal{X}$ gives a fiber bundle:

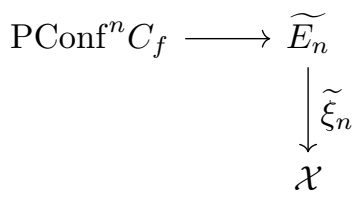

The symmetric group $S_{n}$ acts freely on $\widetilde{E_{n}}$ by permuting the $n$ coordinates. Define $E_{n}:=\widetilde{E_{n}} / S_{n}$. Since the bundle projection $\widetilde{\xi}_{n}: \widetilde{E_{n}} \rightarrow \mathcal{X}$ is invariant of $S_{n}$, it descends to another bundle $\xi_{n}$ : $E_{n} \rightarrow \mathcal{X}$ as in (1.1). The fiber of $\xi_{n}$ over $f \in \mathcal{X}$ is precisely the unordered configuration space of $C_{f}$

$$
\mathrm{UConf}^{n} C_{f}:=\mathrm{PConf}^{n} C_{f} / S_{n} .
$$

Remark 1. The Lie group $\mathrm{PGL}_{3}(\mathbb{C})=\mathrm{GL}_{n}(\mathbb{C}) / \mathbb{C}^{\times}$acts on $\mathbb{C} P^{2}$ by projective linear maps, and thus acts on $\mathcal{X}$ by projective linear change of coordinates. Hence, $\mathrm{PGL}_{3}(\mathbb{C})$ also acts on $\widetilde{E_{n}}$ diagonally; this action projects to a $\mathrm{PGL}_{3}(\mathbb{C})$-action on $E_{n}$. The bundle projection $\xi_{n}: E_{n} \rightarrow \mathcal{X}$ is $\mathrm{PGL}_{3}(\mathbb{C})$-equivariant. However, a continuous section $s$ of the bundle $\xi_{n}$ does not have to be $\mathrm{PGL}_{3}(\mathbb{C})$-equivariant. The $\mathrm{PGL}_{3}(\mathbb{C})$-action on $\mathcal{X}$ will play a key role in the proof of Theorem 2 . 
2.2. Algebraic sections of $\xi_{n}$. Every smooth cubic plane curve $C_{f}$ has

(a) 9 inflection points where the tangent line intersect $C_{f}$ with multiplicity 3 ,

(b) 27 sextatic points where an irreducible conic intersects $C_{f}$ with multiplicity 6 ([2]),

(c) 72 points of type 9 where an irreducible cubic intersects $C_{f}$ with multiplicity 9 ([5]).

More generally, Gattazzo [5] defined points of type $3 k$ on $C_{f}$ to be points where an irreducible curve of degree $k$ intersects $C_{f}$ with multiplicity $3 k$. In particular, inflection points and sextatic points are precisely points of type 3 and 6 , respectively. A proof of the following fact can be found on page 392 in [3]: $3 k$ points $P_{i}$ for $i=1, \ldots, 3 k$ on a cubic curve $C$ are on another curve of degree $k$ if and only if $\sum_{i=1}^{3 k} P_{i}=0$ on $C$ as an elliptic curve with an inflection point as identity. Therefore, points of type $3 k$ on $C$ are precisely the $3 k$-torsion points on $C$ that are not $3 j$-torsion points for any $j<k$, with an inflection point as identity. This fact was also proved in Corollary 4.3 in [5].

Definition 1 (Jordan's 2-totient function). $J_{2}(k)=$ number of elements in $(\mathbb{Z} / k \mathbb{Z})^{2}$ of order $k$.

Jordan's 2-totient function can be computed using the following formula:

$$
J_{2}(k)=k^{2} \prod_{p \mid k, \text { prime }}\left(1-\frac{1}{p^{2}}\right) .
$$

It follows from the discussion above that

$$
9 J_{2}(k)=\text { the number of points of type } 3 k \text { on a cubic curve }
$$

The coordinates of the points of type $3 k$ on $C_{f}$ change continuously as we vary $f$ in the parameter space $\mathcal{X}$. For example, the 9 inflections points are precisely the vanishing locus of the Hessian function, which depends continuously on the defining equation of the cubic curve. Therefore, the points of type $3 k$ define a continuous section of $\xi_{n}$ for $n=9 J_{2}(k)$. More generally, given a finite set of positive integers $S$, the points of type $3 k$ for $k \in S$ define a continuous section of $\xi_{n}$ for $n=9 \sum_{k \in S} J_{2}(k)$, which gives Theorem 1 in the Introduction.

\section{Proof of Theorem 2}

Suppose the bundle $\xi_{n}$ has a continuous section $s: \mathcal{X} \rightarrow E_{n}$.

\section{Step 1: $s$ induces a continuous map $\phi: \mathrm{PGL}_{3}(\mathbb{C}) \rightarrow \mathrm{UConf}^{n} C_{f}$}

Throughout the proof, we will fix a basepoint $f \in \mathcal{X}$ to be the Fermat cubic:

$$
f(x, y, z)=x^{3}+y^{3}+z^{3} .
$$

If we choose a different basepoint in $\mathcal{X}$, the argument will go through with only small modification. See Remark 4 for an explanation. Recall from Section 2 that $\mathrm{PGL}_{3}(\mathbb{C})$ acts on both $E_{n}$ and $\mathcal{X}$. Define a map

$$
\begin{aligned}
\phi: \mathrm{PGL}_{3}(\mathbb{C}) & \rightarrow E_{n} \\
g & \mapsto g \cdot s\left(g^{-1} \cdot f\right)
\end{aligned}
$$

where again $E_{n}$ is the total space of the fiber bundle $\xi_{n}$. Notice that for any $g \in \mathrm{PGL}_{3}(\mathbb{C})$, we have

$$
\begin{aligned}
\xi_{n}(\phi(g)) & =\xi_{n}\left(g \cdot s\left(g^{-1} \cdot f\right)\right) \\
& =g \cdot \xi_{n}\left(s\left(g^{-1} \cdot f\right)\right) \\
& =g \cdot\left(g^{-1} \cdot f\right) \\
& =f .
\end{aligned}
$$

since $\xi_{n}$ is $\mathrm{PGL}_{3}(\mathbb{C})$-equivariant

since $\xi_{n} \circ s=i d_{\mathcal{X}}$

Therefore, the image of $\phi$ is entirely in the fiber of $\xi_{n}$ over $f$. Hence, we will simply consider $\phi$ as a map $\phi: \mathrm{PGL}_{3}(\mathbb{C}) \rightarrow \mathrm{UConf}^{n} C_{f}$. 
Both the domain and the codomain of $\phi$ are acted upon by a finite group $\Gamma_{f}$, the group of projective linear automorphisms of the Fermat cubic curve $C_{f}$ :

$$
\Gamma_{f}:=\left\{g \in \mathrm{PGL}_{3}(\mathbb{C}): g \cdot f=f \in \mathcal{X}\right\} .
$$

$\Gamma_{f}$ acts on $\mathrm{UConf}^{n} C_{f}$ since it acts on the curve $C_{f} . \Gamma_{f}$ is a subgroup of $\mathrm{PGL}_{3}(\mathbb{C})$ and thus acts on $\mathrm{PGL}_{3}(\mathbb{C})$ via multiplication from the left.

Lemma 4. The map $\phi: \mathrm{PGL}_{3}(\mathbb{C}) \rightarrow \mathrm{UConf}^{n} C_{f}$ is $\Gamma_{f}$-equivariant.

Proof. For any $\gamma \in \Gamma_{f}$ and any $g \in \mathrm{PGL}_{3}(\mathbb{C})$, we have

$$
\begin{aligned}
\phi(\gamma g) & =(\gamma g) \cdot s\left(g^{-1} \gamma^{-1} \cdot f\right) \\
& =\gamma \cdot\left(g \cdot s\left(g^{-1} \cdot f\right)\right) \\
& =\gamma \cdot \phi(g)
\end{aligned}
$$

since $\gamma^{-1} \in \Gamma_{f}$ fixes $f$

Observe that $\pi_{1}\left(\mathrm{PGL}_{3}(\mathbb{C})\right) \cong \mathbb{Z} / 3 \mathbb{Z}$ because we have $\mathrm{PGL}_{3}(\mathbb{C}) \cong \mathrm{PSL}_{3}(\mathbb{C})$ which is $\mathrm{SL}_{3}(\mathbb{C})$ modulo third roots of unity. On the other hand, Fadell-Neuwirth (Corollary 2.2 in [4]) proved that the ordered configuration space $\mathrm{PConf}^{n} C_{f}$ (defined in Section 2) is aspherical. The same is true for $\mathrm{UConf}^{n} C_{f}$. So $\pi_{1}\left(\mathrm{UConf}^{n} C_{f}\right)$ must be torsion free because the Eilenberg-MacLane space of any group with torsion must have infinite dimensions. Therefore, $\phi: \mathrm{PGL}_{3}(\mathbb{C}) \rightarrow \mathrm{UConf}^{n} C_{f}$ must induce a trivial map on fundamental groups. By lifting criterion, the map $\phi$ can be lifted to a map $\tilde{\phi}$ making the following diagram commute:

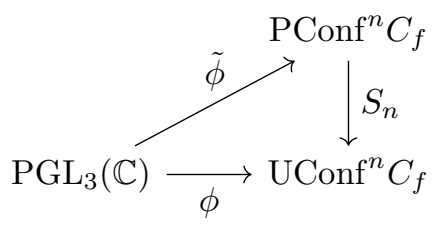

The natural $\Gamma_{f}$-action on $\mathrm{PConf}^{n} C_{f}$ commutes with the $S_{n}$ action.

\section{Step 2: $\phi$ induces a group homomorphism $\rho: \Gamma_{f} \rightarrow S_{n}$.}

The lift $\tilde{\phi}$ may not be $\Gamma_{f}$-equivariant, but it projects down to a $\Gamma_{f}$-equivariant map $\phi$ as in Lemma 4 Therefore, for any $\gamma \in \Gamma_{f}$, there exists a unique permutation $\sigma_{\gamma} \in S_{n}$ such that $\tilde{\phi}(\gamma)=\sigma_{\gamma}(\gamma \cdot \tilde{\phi}(1))$. Let $\rho: \Gamma_{f} \rightarrow S_{n}$ denote the function $\gamma \mapsto \sigma_{\gamma}$.

Lemma 5. (1) For any $\gamma \in \Gamma_{f}$ and any $h \in \operatorname{PGL}_{3}(\mathbb{C})$, we have

$$
\tilde{\phi}(\gamma h)=\sigma_{\gamma}(\gamma \cdot \tilde{\phi}(h)) \text {. }
$$

(2) The function $\rho: \Gamma_{f} \rightarrow S_{n}$ is a group homomorphism.

Proof. (1) Since $\mathrm{PGL}_{3}(\mathbb{C})$ is connected, we can take a path $p:[0,1] \rightarrow \mathrm{PGL}_{3}(\mathbb{C})$ such that $p(0)=1$ and $p(1)=h$. Now $\tilde{\phi}(\gamma \cdot p(t))$ and $\sigma_{\gamma}(\gamma \cdot \tilde{\phi}(p(t)))$ are paths in PConf ${ }^{n} C_{f}$ that both lift the path $\phi(\gamma \cdot p(t))$ in $\mathrm{UConf}^{n} C_{f}$, with the same starting point $\tilde{\phi}(\gamma)=\sigma_{\gamma}(\gamma \cdot \tilde{\phi}(1))$ when $t=0$. Thus they must end at the same point by the uniqueness of path lifting.

(2) Take any $\beta, \gamma \in \Gamma_{f}$. On one hand,

$$
\tilde{\phi}(\beta \gamma)=\sigma_{\beta \gamma}(\beta \gamma \cdot \tilde{\phi}(1)) \text {. }
$$

On the other hand, by 3.1

$$
\tilde{\phi}(\beta \gamma)=\sigma_{\beta}(\beta \cdot \tilde{\phi}(\gamma))=\sigma_{\beta}\left(\beta \cdot \sigma_{\gamma}(\gamma \cdot \tilde{\phi}(1))\right)=\sigma_{\beta} \sigma_{\gamma}(\beta \gamma \cdot \tilde{\phi}(1))
$$

where the last equality follows because the $S_{n}$-action commutes with the $\Gamma_{f}$-action on $\mathrm{PConf}^{n} C_{f}$. Therefore, $\sigma_{\beta} \sigma_{\gamma}=\sigma_{\beta \gamma}$. 
It follows from Lemma 5 that the homomorphism $\rho$ is trivial if and only if the lift $\tilde{\phi}$ is $\Gamma_{f}$ equivariant. Therefore, the group homomorphism $\rho: \Gamma_{f} \rightarrow S_{n}$ measures the failure of the lift $\tilde{\phi}$ to be $\Gamma_{f}$-equivariant.

Lemma 6. Let $\operatorname{Proj}_{i}: \mathrm{PConf}^{n} C_{f} \rightarrow C_{f}$ denote the projection onto the $i$-th coordinate. Consider the action of $\Gamma_{f}$ on $\{1,2, \cdots, n\}$ given by $\rho: \Gamma_{f} \rightarrow S_{n}$. For any $\gamma \in \Gamma_{f}$ and any $i \in\{1,2, \cdots, n\}$, if $\sigma_{\gamma}$ fixes $i$, then the composition $\operatorname{Proj}_{i} \circ \tilde{\phi}$

$$
\mathrm{PGL}_{3}(\mathbb{C}) \stackrel{\tilde{\phi}}{\rightarrow} \mathrm{PConf}^{n} C_{f} \stackrel{\operatorname{Proj}_{i}}{\longrightarrow} C_{f}
$$

is equivariant with respect to $\gamma$.

Proof. Given any $h \in \mathrm{PGL}_{3}(\mathbb{C})$, Lemma 5 gives that $\tilde{\phi}(\gamma \cdot h)=\sigma_{\gamma}(\gamma \cdot \tilde{\phi}(h))$. Thus, whenever $\sigma_{\gamma}(i)=i$, we have

$$
\operatorname{Proj}_{i} \circ \tilde{\phi}(\gamma \cdot h)=\operatorname{Proj}_{i}\left(\sigma_{\gamma}(\gamma \cdot \tilde{\phi}(h))\right)=\operatorname{Proj}_{i}(\gamma \cdot \tilde{\phi}(h))=\gamma \cdot \operatorname{Proj}_{i}(\tilde{\phi}(h)) .
$$

Remark 2 (Interpretation of the group homomorphism $\rho$ as monodromy). The above construction of the group homomorphism $\rho$ from a continuous section $s$ appears to be ad hoc. We now briefly sketch a more conceptual but less direct construction of $\rho$ to illustrate that $\rho$ is a natural object to consider for finding obstructions to sections. We do not need this equivalent construction of $\rho$ anywhere in the proof.

A continuous section $s$ produces a cover of $\mathcal{X}$ of degree $n$, where the fiber over each curve is the set of $n$ points on the curve chosen by $s$. The monodromy representation of this cover gives a group homomorphism

$$
\pi_{1}(\mathcal{X}) \rightarrow S_{n}
$$

By restricting the monodromy representation to the $\mathrm{PGL}_{3}(\mathbb{C})$-orbit of the Fermat curve

$$
\mathrm{PGL}_{3}(\mathbb{C}) / \Gamma_{f} \approx \mathrm{PGL}_{3}(\mathbb{C}) \cdot f \hookrightarrow \mathcal{X}
$$

we obtain a group homomorphism

$$
\pi_{1}\left(\mathrm{PGL}_{3}(\mathbb{C}) / \Gamma_{f}\right) \rightarrow S_{n}
$$

This homomorphism factors through the quotient $\pi_{1}\left(\mathrm{PGL}_{3}(\mathbb{C}) / \Gamma_{f}\right) \rightarrow \Gamma_{f}$, making the following diagram commute:

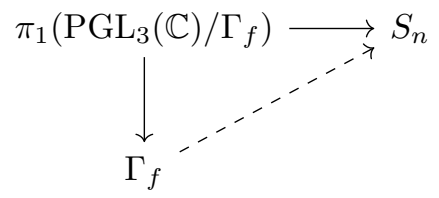

The induced homomorphism $\Gamma_{f} \rightarrow S_{n}$ is conjugate to $\rho$.

Step 3: $\boldsymbol{n}$ must be a multiple of 9. The homomorphism $\rho: \Gamma_{f} \rightarrow S_{n}$ gives an action of $\Gamma_{f}$ on $\{1,2, \ldots, n\}$. In the final step, we will show that $\Gamma_{f}$ contains a subgroup $K$ of order 9 that acts freely. Thus, $\{1,2, \ldots, n\}$ is a disjoint union of orbits of $K$, each of size 9 . In particular, $n$ must be a multiple of 9 .

Lemma 7. There are two commuting elements $a$ and $b$ in $\Gamma_{f}$ such that

(1) the subgroup $K:=\langle a, b\rangle \leq \Gamma_{f}$ is isomorphic to $\mathbb{Z} / 3 \mathbb{Z} \times \mathbb{Z} / 3 \mathbb{Z}$.

(2) $a$ and $b$ act on $C_{f}$ as translations of order 3.

Remark 3. Lemma 7 was mentioned without proof in [1], first paragraph of Section 4. We include a brief proof here to make this paper complete and self-contained. 
Proof. Let $a$ and $b$ be two elements in $\mathrm{PGL}_{3}(\mathbb{C})$ that are represented by the following two matrices:

$$
a=\left[\begin{array}{lll}
0 & 0 & 1 \\
1 & 0 & 0 \\
0 & 1 & 0
\end{array}\right], \quad b=\left[\begin{array}{ccc}
1 & 0 & 1 \\
0 & e^{2 \pi i / 3} & 0 \\
0 & 0 & e^{4 \pi i / 3}
\end{array}\right] .
$$

The commutator of the two matrices is in the center of $\mathrm{GL}_{3}(\mathbb{C})$. Thus, $a$ and $b$ commute in $\mathrm{PGL}_{3}(\mathbb{C})$. Both elements are of order 3 and preserve the Fermat cubic $f: x^{3}+y^{3}+z^{3}$. Thus, (1) is established.

We first claim that $a$ acts on $C_{f}$ via translation. In general, every morphism of an elliptic curve is a composition of a translation and a group homomorphism. Thus, it suffices to show that the induced map $a_{*} \in \operatorname{Aut}\left(H_{1}\left(C_{f}, \mathbb{Z}\right)\right)=\mathrm{SL}_{2}(\mathbb{Z})$ is identity. A straightforward computation shows that the automorphism $a$ on $C_{f}$ does not have any fixed point. Thus, by the Lefschetz fixed point theorem, $a_{*}$ must have trace 2 . Now $a_{*}$ is a finite order element in $\mathrm{SL}_{2}(\mathbb{Z})$ of trace 2 , and therefore must be identity.

The exact same argument applies to $b$.

Lemma 8. For any $\gamma \in K=\langle a, b\rangle$, if there is a continuous $\gamma$-equivariant map $\psi: \mathrm{PGL}_{3}(\mathbb{C}) \rightarrow C_{f}$, then $\gamma$ must be the identity.

Proof. Note that $\psi: \mathrm{PGL}_{3}(\mathbb{C}) \rightarrow C_{f}$ must induce a trivial map on fundamental groups since $\pi_{1}\left(\mathrm{PGL}_{3}(\mathbb{C})\right) \cong \mathbb{Z} / 3 \mathbb{Z}$ and $\pi_{1}\left(C_{f}\right) \cong \mathbb{Z}^{2}$. Thus we can lift $\psi$ to $\tilde{\psi}$ making the following diagram commute:

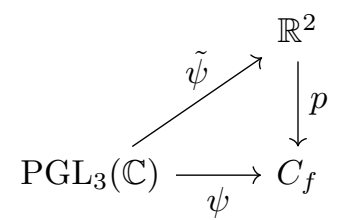

$\gamma \in K$ is a translation of the torus $C_{f}$. Let $\tilde{\gamma}: \mathbb{R}^{2} \rightarrow \mathbb{R}^{2}$ be the unique translation of $\mathbb{R}^{2}$ such that

$$
\tilde{\gamma}(\tilde{\psi}(1))=\tilde{\psi}(\gamma)
$$

We claim that for any $h \in \mathrm{PGL}_{3}(\mathbb{C})$,

$$
\tilde{\gamma}(\tilde{\psi}(h))=\tilde{\psi}(\gamma h) .
$$

Take a path $\mu(t)$ in $\mathrm{PGL}_{3}(\mathbb{C})$ such that $\mu(0)=1$ and $\mu(1)=h$. The two paths $\tilde{\gamma}(\tilde{\psi}(\mu(t))$ and $\tilde{\psi}(\gamma \cdot \mu(t))$ are both lifts of the path $\gamma(\psi(\mu(t)))=\psi(\gamma \cdot \mu(t))$ starting at the same point $\tilde{\gamma}(\tilde{\psi}(1))=\tilde{\psi}(\gamma)$. Thus they must end at the same point by the uniqueness of path lifting, giving that $\tilde{\gamma}(\tilde{\psi}(h))=\tilde{\psi}(\gamma h)$.

Applying (3.2) three times, we have

$$
\tilde{\gamma}^{3}(\tilde{\psi}(1))=\tilde{\psi}\left(\gamma^{3}\right)=\tilde{\psi}(1) .
$$

Thus, $\tilde{\gamma}^{3}$ must be the trivial translation of $\mathbb{R}^{2}$, and so is $\tilde{\gamma}$. In this case (3.2) gives that $\tilde{\psi}(1)=\tilde{\psi}(\gamma)$, and thus $\psi(1)=\psi(\gamma)=\gamma \cdot \psi(1)$. $\gamma$ is a translation of the torus $C_{f}$ that has a fixed point $\psi(1)$, and therefore must be the identity map.

Recall that the group homomorphism $\rho: \Gamma_{f} \rightarrow S_{n}$ gives an action of $\Gamma_{f}$ on $\{1,2, \cdots, n\}$. Now Lemma 6] and Lemma 8 together imply that the subgroup $K \leq \Gamma_{f}$ acts freely on $\{1,2, \cdots, n\}$. Thus, $\{1,2, \cdots, n\}$ decomposes to a disjoint union of $K$-orbits of size 9 . In particular, $n$ must be a multiple of 9 . This concludes the proof of Theorem 2 .

Remark 4 (What if we choose a different basepoint?). The choice of the Fermat cubic curve $f(x, y, z)=x^{3}+y^{3}+z^{3}$ as a basepoint of $\mathcal{X}$ allows us to explicitly write down matrices for $a$ and $b$ in Lemma 7 . If a different basepoint $h \in \mathcal{X}$ is chosen, the argument will go through 
with only minor modification. There is an element $g \in \mathrm{PGL}_{3}(\mathbb{C})$ that brings $h$ to be in the Hesse form: $x^{3}+y^{3}+z^{3}+\lambda x y z$ for some $\lambda \in \mathbb{C}$ (see e.g. Lemma 1 in [1]). One can check that $a$ and $b$ constructed in Lemma 7 act as translations on any smooth cubic curve in the Hesse form. Thus $g a g^{-1}$ and $g b g^{-1}$ act on $C_{h}$ as translations. Now the argument goes through by replacing $a$ and $b$ by their $g$-conjugates.

\section{REFERENCES}

[1] M. Artebani and I. Dolgachev, The Hesse pencil of cubic plane curves, Enseign. Math., 55, 3/4 (2009), $235-273$.

[2] A. Cayley, On the Sextactic Points of a Plane Curve, Philos. Trans. R. Soc. Lond. 155 (1865), 545-578.

[3] A. Clebsch: Leçons sur la géométrie, tome II, Paris 1880.

[4] E. Fadell and L. Neuwirth, Configuration spaces Math. Scand. 10 (1962), 111-118.

[5] R. Gattazzo, Points of type 9 on an elliptic cubic. Rend. Sem. Mat. Univ. Padova 61 (1979), 285-301. 